\title{
Projecting the Short-Term Trend of COVID-19 in Iraq
}

\author{
MURTADHA ALDEER, WINLAB, Rutgers University, USA \\ AHMED AL HILLI, Al-Furat Al-Awsat Technical University, Iraq \\ ISSAM S. ISMAIL, Wassit Health Directorate, Iraq
}

\begin{abstract}
The novel coronavirus that causes the now well-known COVID-19 illness has affected the whole world as the number of daily reported cases has exceeded 250,000. The spread trend of the virus across the globe has varied from one country to another, mainly because of each government's response. In this article, we discuss the statistics of the cases in Iraq and provide a projection model based on the current situation and government interventions. Using daily reported cases data from February 24, 2020-July 31, 2020, we report 6-week-ahead projections of daily cases, critical cases, and deaths. Specifically, we propose a simple machine learning-based model based on Gaussian Process regression to project the future trend of COVID-19 in Iraq. This work aims to aid the Iraqi government by providing a statistical tool to provide future estimates of the suspected and death cases resulting from COVID-19 and make strategic decisions.
\end{abstract}

CCS Concepts: • Computing methodologies $\rightarrow$ Model verification and validation; Machine learning approaches;

Additional Key Words and Phrases: Iraq, COVID-19, machine learning

ACM Reference format:

Murtadha Aldeer, Ahmed Al Hilli, and Issam S. Ismail. 2020. Projecting the Short-Term Trend of COVID-19 in Iraq. Digit. Gov.: Res. Pract. 2, 1, Article 14 (December 2020), 7 pages.

https://doi.org/10.1145/3431769

\section{INTRODUCTION}

The world is facing a historical challenge due to the COVID-19 pandemic caused by the spread of the novel coronavirus. Since its start in China, the virus has continued to spread across the globe causing more people to become sick and more casualties. Even though the virus hit almost the whole globe, there are countries that have been hit harder than others. As of July, 31, 2020, there have been $>17.5$ million confirmed cases and $>679,000$ deaths worldwide [JHU 2020].

As in many other parts of the world, COVID-19 was visible in the Middle East region. Within that region, Iraq is a country with an estimated population of 38 million citizens. It is surrounded by six countries, including Iran, one of the top countries hit by the coronavirus disease [Statista 2020]. On February 19, 2020, the first two individuals with confirmed COVID-19 were reported in Iran [Arab-Mazar et al. 2020]. On February 24, 2020,

On leave from the Ministry of Communications, Basrah, Iraq.

Authors' addresses: M. Aldeer (corresponding author), WINLAB, Rutgers University, 671 US-1 South, North Brunswick, NJ, 08902, USA; email: maldeer@winlab.rutgers.edu; A. Al Hilli (corresponding author), Al-Furat Al-Awsat Technical University, Najaf, Iraq; email: ahmed.alhilli@atu.edu.iq; I. S. Ismail, Wassit Health Directorate, Wassit, Iraq; email: issam_health@yahoo.com.

Permission to make digital or hard copies of all or part of this work for personal or classroom use is granted without fee provided that copies are not made or distributed for profit or commercial advantage and that copies bear this notice and the full citation on the first page. Copyrights for components of this work owned by others than ACM must be honored. Abstracting with credit is permitted. To copy otherwise, or republish, to post on servers or to redistribute to lists, requires prior specific permission and/or a fee. Request permissions from permissions@acm.org.

(c) 2020 Association for Computing Machinery.

2639-0175/2020/12-ART14 \$15.00

https://doi.org/10.1145/3431769

Digital Government: Research and Practice, Vol. 2, No. 1, Article 14. Publication date: December 2020. 


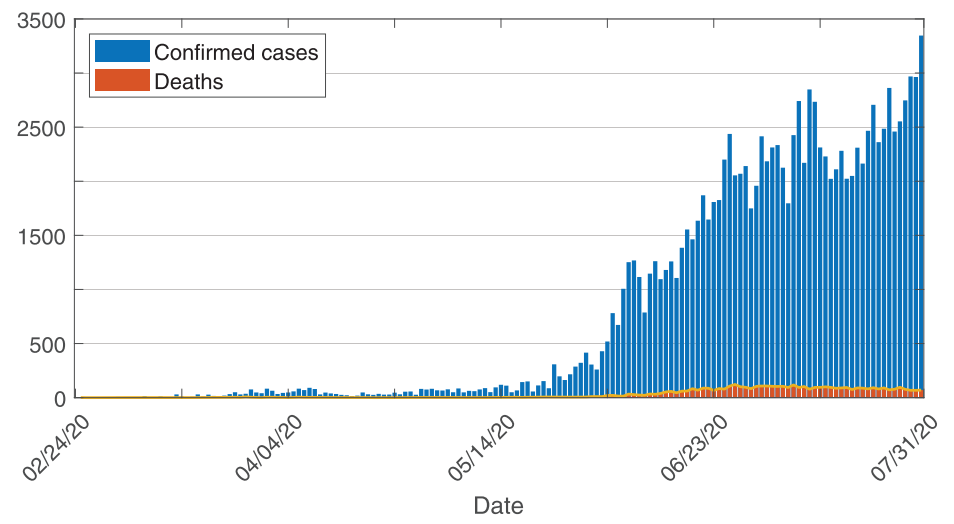

Fig. 1. Number of confirmed cases and deaths related to COVID-19 in Iraq for the period February 24, 2020-July 31, 2020 (source: Dong et al. [2020]).

Iraq reported the first individual with confirmed COVID-19 [Mikhael and Al-Jumaili 2020]. As of July, 31, 2020, there are more than 124,000 cumulative confirmed cases and $>4,700$ cumulative deaths related to COVID-19 in Iraq (Figure 1Figure 1). The vulnerability of an outbreak is still high in Iraq due to several challenges-including inadequate awareness, the capacities of quarantine facilities, a shortage of personal protective equipment, and inadequate hygiene [Mikhael and Al-Jumaili 2020]. The Iraqi government provides daily briefings about the new COVID-19-related cases and deaths. However, to the best of our knowledge, there is no published work that proposes a data tracking model to track and predict future COVID-19 cases and deaths in Iraq. Despite that, Iraq is witnessing a notable increase in COVID-19-related cases.

Data analytic and machine learning are useful tools that have proven to accelerate COVID-19-related tasks such as diagnosis and treatment, contact tracing, prediction and forecasting, and decision making [Lalmuanawma et al. 2020; Peiffer-Smadja et al. 2020; Wang et al. 2020]. In this work, we propose a machine learning approach for projecting the expected number of COVID-19-related cases in Iraq, under certain circumstances, for the upcoming weeks. We believe that the proposed model can help to provide a figure of the expected COVID19 cases in the near future and thus allow the Iraqi government to fight the pandemic. The rest of this article is organized as follows. Our prediction methodology is discussed in Section 2. Our experimental results are depicted and discussed in Section 3. Finally, Section 4 provides our conclusions.

\section{METHODOLOGY}

COVID-19 spread depends on many factors-including social distancing, awareness, and governmental response. The scarcity of such measures in Iraq makes it difficult to include these factors in data modeling approaches. We construct a statistical forecasting model that uses COVID-19 cases data from the past and uses it to provide shortterm predictions. The framework of our model is mainly based on the Gaussian Process (GP) as a machine learning predictor. The GP is a non-parametric modeling approach that can provide short-term prediction based only on observations. It builds its prediction based on the interaction among training data, without explicitly providing the affecting factors. In this section, we discuss the mathematical and machine learning tools used to predict the trend of COVID-19 cases in Iraq. Also, we define the predicted statistics and introduce the data we used.

\subsection{Gaussian Process}

The GP [Liu et al. 2020] is a stochastic process (usually indexed by time or space) in which any subset of observations follow the multivariate Gaussian Distribution. Given $n$ observations $\left\{y_{1}, y_{2}, \ldots y_{n}\right\}$ along with their indices $\left\{x_{1}, x_{2}, \ldots, x_{n}\right\}$, the distribution $p\left(y_{1}, y_{2}, \ldots, y_{n}\right)$ is a multivariate Gaussian. Multivariate Gaussian distribution 
can be fully described by two parameters, its mean (which is usually assumed to be zero) and its co-variance matrix (which is usually called the kernel), which plays an important role on the behavior of GP. In machine learning, GP finds application in regression, prediction, classification, and even dimentionality reduction. Given a set of $n$ training points $\left\{\left(x_{1}, y_{1}\right),\left(x_{2}, y_{2}\right), \ldots,\left(x_{n}, y_{n}\right)\right.$, the kernel matrix can be calculated as

$$
\mathbf{K}=\left[\begin{array}{cccc}
k\left(x_{1}, x_{1}\right) & k\left(x_{1}, x_{2}\right) & \ldots & k\left(x_{1}, x_{n}\right) \\
k\left(x_{2}, x_{1}\right) & k\left(x_{2}, x_{2}\right) & \ldots & k\left(x_{2}, x_{n}\right) \\
\vdots & \vdots & \ddots & \vdots \\
k\left(x_{n}, x_{1}\right) & k\left(x_{n}, x_{2}\right) & \ldots & k\left(x_{n}, x_{n}\right)
\end{array}\right]
$$

where $k\left(x_{i}, x_{j}\right)$ is the kernel function between $x_{i}$ and $x_{j}$. In prediction, given a new space or time point $x_{*}$, we want to find its corresponding prediction $y_{*}$. Following the assumption in GP that states that any subset of points follow multivariate Gaussian distribution, we can write

$$
\left[\begin{array}{c}
\mathbf{y} \\
y_{*}
\end{array}\right] \sim \mathcal{N}\left(\mathbf{0},\left[\begin{array}{cc}
\mathbf{K} & \mathbf{k}_{*}^{T} \\
\mathbf{k}_{*} & k_{* *}
\end{array}\right]\right)
$$

where $\mathbf{k}_{*}=\left[k\left(x_{*}, x_{1}\right) k\left(x_{*}, x_{2}\right) \ldots k\left(x_{*}, x_{n}\right)\right]$ and $k_{* *}=k\left(x_{*}, x_{*}\right)$. From Equation (2), the probability distribution function of $y_{*}$ given $\mathbf{y}$ can be written as

$$
y_{*} \mid \mathbf{y} \sim \mathcal{N}\left(\mathbf{k}_{*} \mathbf{K}^{-1} \mathbf{y}, k_{* *}-\mathbf{k}_{*} \mathbf{K}^{-1} \mathbf{k}_{*}{ }^{T}\right)
$$

and the maximum likelihood estimated of Equation (3) for $y_{*}$ is

$$
\hat{y}_{*}=\mathbf{k}_{*} \mathbf{K}^{-1} \mathbf{y}
$$

with variance

$$
\operatorname{var}\left(\hat{y}_{*}\right)=k_{* *}-\mathbf{k}_{*} \mathbf{K}^{-1} \mathbf{k}_{*}{ }^{T} .
$$

The above procedure can be repeated for any time or space new points to predict their corresponding values. As mentioned above, the kernel function plays an important role in estimating the final values in Equation (4) and Equation (5). Since we do not expect big variation across the confirmed cases in small time interval, and since the current confirmed cases shows correlation with previous and most probably future cases, we choose square exponential as a kernel function in our model. Square exponential kernel function are one of the most widely used kernel function in GP. It samples for smooth functions with correlation among near by samples. Mathematically, square exponential can be described as

$$
k\left(x_{i}, x_{j}\right)=\sigma_{f}^{2} e^{\frac{-\left(x_{i}-x_{j}\right)^{2}}{2 l^{2}}},
$$

where $\sigma_{f}$ is the maximum variance that can be reached and $l$ represents the length scale. This method does not require heavy computation for short-term forecasting, and thus processing time is very short. Note that this is promising for running the proposed framework on the web to provide daily trends upon the availability of new data.

\subsection{Projected Statistics}

Continuously predicting the course of the COVID-19 epidemic can help in addressing different questions on different timescales. In the context of Iraq, we use the following statistics in our study, as they are widely used in COVID-19-related studies [Hoseinpour Dehkordi et al. 2020; Max et al. 2020].

- Cases of COVID-19: This is the number of confirmed cases of patients that have coronavirus predicted per day.

- Deaths from COVID-19: This is the number of deaths among COVID-19 cases per day.

- Critical cases of COVID-19: This is the predicted number of COVID-19 patients that need intensive care.

Digital Government: Research and Practice, Vol. 2, No. 1, Article 14. Publication date: December 2020. 
Table 1. Experimental Settings

\begin{tabular}{|c|c|}
\hline Parameter & Value \\
\hline \hline$\sigma$ & 48.464 \\
\hline$l$ & 60 \\
\hline Total number of days in the dataset & 158 days \\
\hline Training sample size (number of days used for training) & 142 days \\
\hline Testing sample size (number of days used for testing) & 16 days \\
\hline Projection period & 6 weeks \\
\hline
\end{tabular}

Forecasting the number of confirmed cases and deaths allows the government to make critical decisions (e.g., strict social distancing, quarantine, travel advisory, border closure, and curfews), which have been proven to slow down the spread of corona virus and save lives [Pearce 2020]. Meanwhile, the critical cases are the individuals who need extensive care. Thus, predicting the number of critical cases is also important, since it provides the number of Intensive Care Unit beds required.

\subsection{Data}

We obtained data on the confirmed and death cases reported in Iraq covering the period February 24 to July 31, 2020, from the Johns Hopkins Coronavirus Resource Center [Dong et al. 2020; JHU 2020]. The dataset includes the number of confirmed and death cases by the end of each day. The dataset preserves the temporal components, as it uses a time-series format with date, month, and year. To project the daily number of future critical cases, we manually collected the data of past cases from the daily announcements available on the Iraqi Ministry of Health website [MOH-Iraq 2020]. Because of the sparse record and the lack of detailed history of the number of critical cases in Iraq, the trend of the critical cases curve is just a scaled-down version of the confirmed cases curve. The scale is calculated as the average of the ratios of the critical cases to the number of confirmed cases in the dates that such record was found. Other parameters, such as wearing masks and social-distancing, were not considered, as it is difficult to obtain statistics about them.

\section{RESULTS AND DISCUSSION}

\subsection{Results}

In this section, we present the results of our model and our prediction regarding the number of cases in the next 6 weeks. The results and discussion are based on the time series of the number of confirmed cases starting from Febrary 24, 2020, to July 26, 2020. The available time series is first divided into two subsets: training and testing subsets. The time indices along with their corresponding confirmed cases for the training subset were chosen randomly. The length of the training subset is set to be $90 \%$ of the length of the whole time series. The remaining $10 \%$ of the data was used in the testing phase. In training phase, the training subset was used to calculate kernel matrix as in Equation (1). The square exponential kernel was used, and the values of $\sigma_{f}$ and $l$ were estimated using grid search. Using the kernel matrix, and for all data in the training subset, Equation (4) was used to estimate the mean number of confirmed cases, deaths, and critical cases, respectively. To estimated the lower and upper bounds for the prediction error for our GP model, we use the variance as a prediction error measure [Beckers et al. 2018]. Our experimental settings are outlined in Table 1.

Figure 2Figure 2 shows the history and the projection of the daily cases. The grey line represents the training samples, while the red dots represent the testing samples. To create a baseline and to validate our approach on the actual data, we compute the predicted response for the test data (and associated standard deviation), hence the green curve. It evident that the difference between the predicted cases and the baseline cases is small. Also,

Digital Government: Research and Practice, Vol. 2, No. 1, Article 14. Publication date: December 2020. 


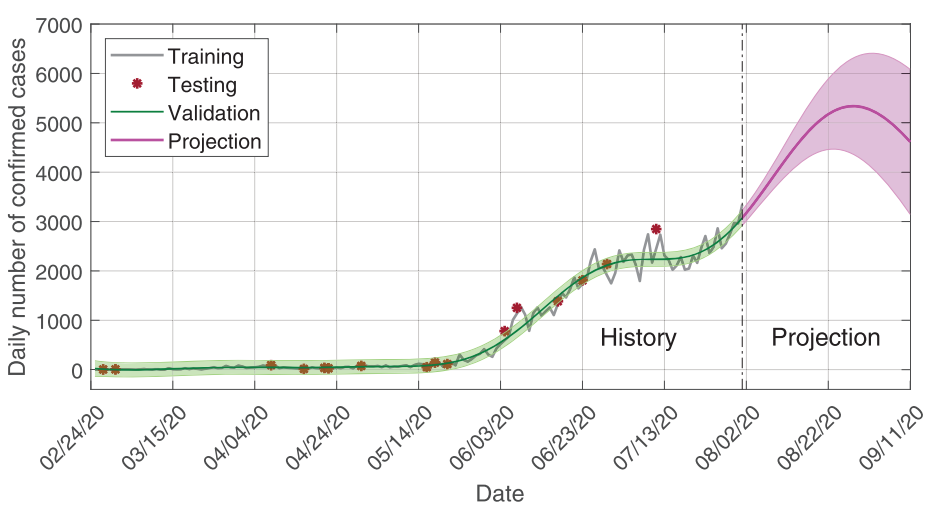

Fig. 2. Predicted number of new cases.

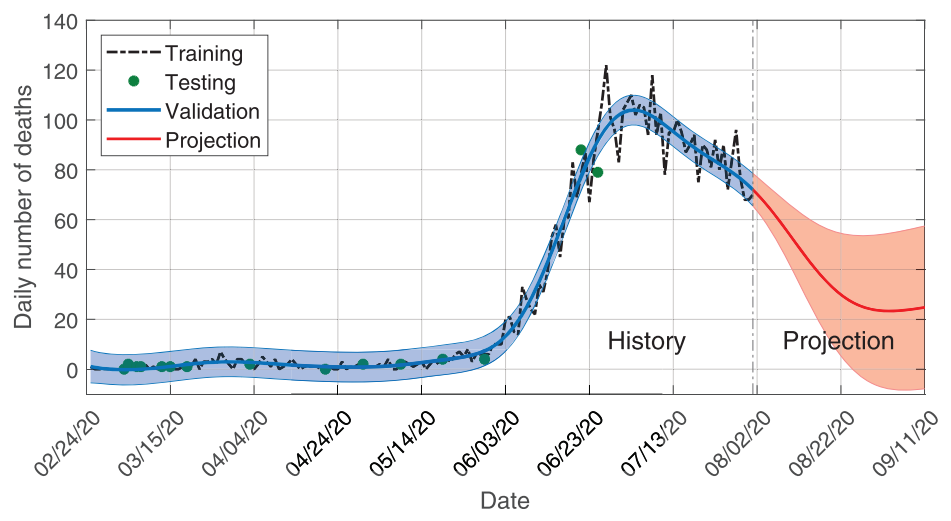

Fig. 3. Predicted number of deaths.

we conduct a prediction based on the available time series to project the average number of cases (along with its variance) in the next 6 weeks, August 1, 2020-September 11, 2020, (the pink curve). According to our model, the number of cases will increase before the curve starts to bend, assuming that the affecting features remain the same.

The same analysis described earlier in this section was conducted to project the number of deaths for the next 6 weeks, which is shown in Figure 3Figure 3. The black dashed line represents the training samples, while the green dots represent the testing samples. We compute the predicted response (as a baseline) for the test data, and that is shown in the blue curve. The predicted average number of deaths and its variance in the next 6 weeks, starting from August 1, 2020, is illustrated in the orange curve. Fortunately, according to our model and based on the history of death cases, the number is decreasing and will continue to decrease in the next 6 weeks. This can be attributed to using the convalescent plasma therapy [Abdullah et al. 2020; Rasheed et al. 2020] that was adopted by the Iraqi Ministry of Health as a treatment protocol for COVID-19. This is expected, as the recent days showed a decline in the confirmed COVID-19 deaths in Iraq.

In Figure 4Figure 4, we present the trend of the critical cases. It can be seen that the trend in critical cases follows the same pattern of the confirmed cases. This is anticipated, since the critical cases number is a scalar factor of the confirmed cases.

It is worth mentioning that these projections remain uncertain as impacting factors may change. These factors include improvement in clinical skills for managing critical cases, weakness of virus virulence, infection decline 


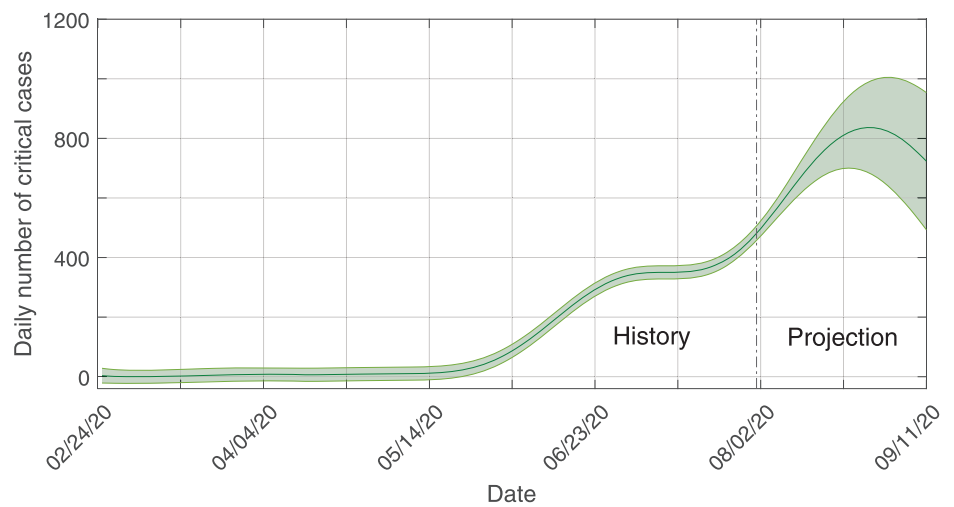

Fig. 4. Predicted number of critical cases.

among vulnerable groups (patients with chronic diseases), and improving the diagnosis/differential diagnosis of causes of death.

\subsection{Discussion}

In this section, we briefly discuss some challenges that face Iraq at this critical time. Due to the special social and economic conditions in Iraq, administrators did not implement a complete lock-down strategy. Also, at the time of writing, protests are taking place in different parts of Iraq demanding jobs and essential services [Mansour et al. 2020]. Nonetheless, in such congregations, social distancing cannot be exercised. However, a strict national lockdown strategy and social distancing measures have proven to eliminate the harm that COVID-19 poses to lives in other parts of the globe [Cousins 2020; Wilson 2020]. Furthermore, to track the pandemic trend as accurately as possible, essential planning and response technologies should be present-including testing, contact tracing, strict quarantine, and access to health-care [Whitelaw et al. 2020]. Some of these technologies have not been implemented in the context of Iraq. One takeaway from this study is the need to use data and other technologies to mitigate the spread of COVID-19 in a country that is already facing many challenges in other life sectors [Aldeer et al. 2016].

\section{CONCLUSIONS}

In this article, a machine learning model based on the Gaussian Process is used to obtain qualitative projections about the mean of the possible number of daily COVID-19 suspected cases and deaths in Iraq. Given that Iraq has not used any previous data-driven approach to fight the COVID-19 pandemic, the estimated number of projected cases will be a beneficial tool for policymakers and health officials. This projection will aid in making necessary allocations for necessary resources and supplies, such as beds, ventilators, and medications. As a result, it can help the government to gain control over the COVID-19 outbreak. Looking forward, we will develop an interactive web-based system that runs our light computation machine learning model and provides projections in real time as new data are updated on a daily basis.

\section{REFERENCES}

Hadi Mohammed Abdullah, Hersh H. Hama-Ali, Sabah Nasraddin Ahmed, Kosar Muhammad Ali, Kamaran Amin Karadakhy, Safeen Othman Mahmood, Zana Hameed Mahmood, Karmand Qadir Hamad Amin, Peshnyar Muhammad Atta, Bryar Ezadeen Nuradeen, et al. 2020. Severe refractory COVID-19 patients responding to convalescent plasma; A case series. Ann. Med. Surg. 56 (August 2020), 125-127.

Murtadha M. N. Aldeer, Richard E. Howard, Richard P. Martin, Khalil Alkadhimi, and Latifah M. Kamarudin. 2016. Towards harnessing Wireless Sensor Networks for supporting the development process in Iraq. In Proceedings of the 2016 IEEE Conference on Open Systems (ICOS'16). 81-86.

Digital Government: Research and Practice, Vol. 2, No. 1, Article 14. Publication date: December 2020. 
Zahra Arab-Mazar, Ranjit Sah, Ali A. Rabaan, Kuldeep Dhama, and Alfonso J. Rodriguez-Morales. 2020. Mapping the incidence of the COVID19 hotspot in Iran-Implications for Travellers. Travel Med. Infect. Dis. 34 (March-April 2020), 101630.

Thomas Beckers, Jonas Umlauft, and Sandra Hirche. 2018. Mean square prediction error of misspecified Gaussian process models. In Proceedings of the 2018 IEEE Conference on Decision and Control (CDC'18). 1162-1167.

Sophie Cousins. 2020. New Zealand eliminates COVID-19. The Lancet 395, 10235 (2020), 1474.

Ensheng Dong, Hongru Du, and Lauren Gardner. 2020. An interactive web-based dashboard to track COVID-19 in real time. Lancet Infect. Dis. 20, 5 (2020), 533-534.

Amirhoshang Hoseinpour Dehkordi, Majid Alizadeh, Pegah Derakhshan, Peyman Babazadeh, and Arash Jahandideh. 2020. Understanding epidemic data and statistics: A case study of COVID-19. 7. Med. Virol. 92, 7 (2020), 868-882.

JHU. 2020. Johns Hopkins Coronavirus Resource Center-Global Map. Retrieved July 25, 2020 from https://coronavirus.jhu.edu/.

Samuel Lalmuanawma, Jamal Hussain, and Lalrinfela Chhakchhuak. 2020. Applications of machine learning and artificial intelligence for Covid-19 (SARS-CoV-2) pandemic: A review. Chaos Solitons Fract. 139 (October 2020), 110059.

H. Liu, Y. Ong, X. Shen, and J. Cai. 2020. When Gaussian process meets big data: A review of scalable GPs. IEEE Trans. Neural Netw. Learn. Syst. (2020), 1-19. DOI : https://doi.org/10.1109/TNNLS.2019.2957109

Wael Mansour, Ashwaq Natiq Maseeh, Bledi Celiku, Alexandre Hugo Laure, Marolla Haddad, and Zoe Cordelia Lu. 2020. Iraq Economic Monitor: Navigating the Perfect Storm (Redux). Technical Report. The World Bank.

Roser Max, Ritchie Hannah, Ortiz-Ospina Esteban, and Joe Hasell. 2020. Coronavirus Disease (COVID-19) eStatistics and Research. Retrieved July 31, 2020 from https://ourworldindata.org/coronavirus.

Ehab Mudher Mikhael and Ali Azeez Al-Jumaili. 2020. Can developing countries alone face corona virus? An Iraqi situation. Publ. Health Pract. 1 (November 2020), 100004.

MOH-Iraq. 2020. Iraqi Ministry of Health. Retrieved July 31, 2020 from https://moh.gov.iq/.

Katie Pearce. 2020. What is social distancing and how can it slow the spread of COVID-19. The Hub (2020). Retrieved November 12, 2020 from https://hub.jhu.edu/2020/03/13/what-is-social-distancing/.

Nathan Peiffer-Smadja, Redwan Maatoug, François-Xavier Lescure, Eric D’Ortenzio, Joëlle Pineau, and Jean-Rémi King. 2020. Machine learning for COVID-19 needs global collaboration and data-sharing. Nat. Mach. Intell. 2 (2020), 293-294.

Anwar M. Rasheed, Dhurgham F. Ftak, Hashim A. Hashim, Mohammed F. Maulood, Khulood K. Kabah, Yaqoob A. Almusawi, and Ahmed S. Abdulamir. 2020. The therapeutic effectiveness of Convalescent plasma therapy on treating COVID-19 patients residing in respiratory care units in hospitals in Baghdad, Iraq. medRxiv (2020). DOI : https://doi.org/10.1101/2020.06.24.20121905

Statista. 2020. Number and change of coronavirus (COVID-19) cases and deaths among the most impacted countries worldwide as of July 27, 2020. Retrieved July 31, 2020 from https://www.statista.com/statistics/1105264/coronavirus-covid-19-cases-most-affected-countriesworldwide/.

C. Jason Wang, Chun Y. Ng, and Robert H. Brook. 2020. Response to COVID-19 in Taiwan: Big data analytics, new technology, and proactive testing. J. Am. Med. Assoc. 323, 14 (2020), 1341-1342.

Sera Whitelaw, Mamas A. Mamas, Eric Topol, and Harriette G. C. Van Spall. 2020. Applications of digital technology in COVID-19 pandemic planning and response. Lancet Dig. Health (2020).

Suze Wilson. 2020. Pandemic leadership: Lessons from New Zealand's approach to COVID-19. Leadership 16, 3 (2020), 279-293.

Received August 2020; revised August 2020; accepted October 2020

Digital Government: Research and Practice, Vol. 2, No. 1, Article 14. Publication date: December 2020. 